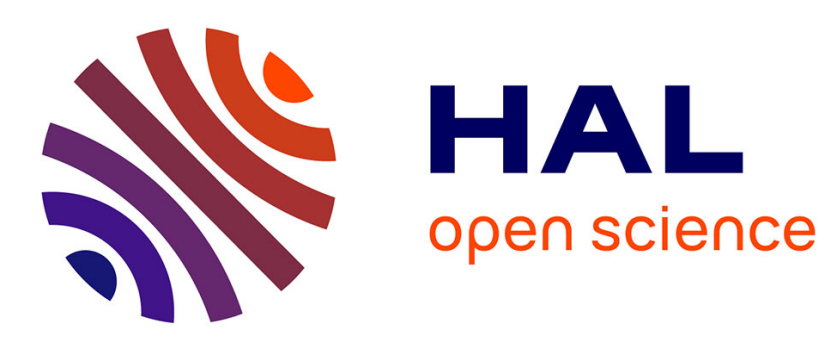

\title{
L'évolution des modalités de décision dans le domaine forestier (Introduction)
}

\author{
Gérard Buttoud, Jean-Claude Monin
}

\section{To cite this version:}

Gérard Buttoud, Jean-Claude Monin. L'évolution des modalités de décision dans le domaine forestier (Introduction). Revue forestière française, 2007, 59 (5), pp.437-442. 10.4267/2042/14865 . hal03449815

\section{HAL Id: hal-03449815 https://hal.science/hal-03449815}

Submitted on 25 Nov 2021

HAL is a multi-disciplinary open access archive for the deposit and dissemination of scientific research documents, whether they are published or not. The documents may come from teaching and research institutions in France or abroad, or from public or private research centers.
L'archive ouverte pluridisciplinaire HAL, est destinée au dépôt et à la diffusion de documents scientifiques de niveau recherche, publiés ou non, émanant des établissements d'enseignement et de recherche français ou étrangers, des laboratoires publics ou privés. 


\section{INTRODUCTION}

\section{L’ÉVOLUTION DES MODALITÉS DE DÉCISION DANS LE DOMAINE FORESTIER}

Depuis une quinzaine d'années, et quelques grandes conférences internationales qui ont mis sur le devant de la scène la gestion durable des forêts, des changements importants sont apparus dans la façon avec laquelle les spécialistes et la société comprennent le rôle de la forêt. $\mathrm{Au}$ contact d'expériences étrangères, grâce à un système d'apprentissage mutuel dont on ne sous-estime plus l'importance, les décisions forestières, aux niveaux global et local, sont en train de changer fortement.

C'est à la compréhension de ces changements qu'un séminaire de réflexion, suscité par le Laboratoire de Politique forestière d'AgroParisTech et financé par le Conseil général des Vosges, a été consacré du 12 au 15 juin 2007, à Tignécourt, commune où ce département possède une forêt dont elle veut faire une référence. À cette occasion, une vingtaine de spécialistes francophones parmi les meilleurs en matière de gestion et de politique forestières, aussi bien scientifiques que gestionnaires, décideurs publics ou privés, élus locaux et représentants d'organisations locales et de défense de l'environnement, ont confronté leurs approches des changements en cours.

La plupart des communications présentées lors de ce séminaire, améliorées suite au contenu de la discussion, forment ce numéro thématique de la Revue forestière française consacré aux modes de gestion et de gouvernance en forêt.

\section{CHANGEMENT ET GOUVERNANCE : LES MOTS CLÉS EN MATIÈRE DE GESTION ET POLITIQUE}

Les principes sur lesquels se basent les politiques en général sont aujourd'hui définis de façon plus ou moins consensuelle dans de grandes conférences internationales dont les recommandations sont signées par les États. Bien sûr ces recommandations n'ont pas force de loi, elles ne s'imposent pas aux nations, mais la pression morale qui s'exerce au niveau international pour partager des valeurs universelles (la démocratie, la paix, la liberté, la préservation de l'environnement, le bien-être des populations, etc.) est telle qu'il est devenu extrêmement rare que les États ne tiennent pas compte de ce qui se définit au niveau mondial.

La forêt ne fait pas exception à cette règle (cf. l'article d'Alain Chaudron).

C'est dire que les principes d'orientation des politiques forestières nationales, de même que les critères et indicateurs qui sont utilisés pour leur suivi et évaluation, sont définis à l'échelle internationale, et s'imposent finalement progressivement à tous ( $c f$. les schémas de certification par exemple).

Les politiques et programmes forestiers établis aux niveaux national, régional et départemental, dépendent donc directement de ces grandes orientations. 
Les orientations internationales données aux politiques et programmes forestiers nationaux et régionaux en ressortent fort différentes des axes en vigueur dans un passé pas si ancien. Au niveau international, les États perdent de leur importance, et apparaissent porteurs d'enjeux non neutres. Par contre, le marché s'est imposé partout comme la référence de base, depuis l'effondrement de l'Union soviétique et la chute du mur de Berlin. Les idéaux partagés à l'échelle du monde ne donnent plus aux États un rôle déterminant dans les orientations politiques, mais laissent en fin de compte au marché la responsabilité de l'allocation des ressources.

Cette évolution est particulièrement nette dans les pays soumis à une forte pression internationale, par exemple les pays tropicaux dépendant fortement des stratégies des bailleurs de fonds (cf. l'article de Robert Nasi et Stéphane Guéneau). C'est le plus souvent à leur sujet ou sur leur exemple que de nouveaux instruments susceptibles de promouvoir la gestion durable des forêts sans nécessairement recourir à la réglementation ont été élaborés (cf. les articles de Stéphane Guéneau, et d'Alain Karsenty \& Romain Pirard).

Le terme, aujourd'hui à la mode, de "gouvernance", est utilisé pour caractériser ces évolutions (cf. l'article introductif de Gérard Buttoud). En opposition au système de décision publique traditionnel donnant aux États un pouvoir absolu et fait de règlements imposés aux assujettis - les Anglo-Saxons emploient pour caractériser ce système le mot de "gouvernement" (government) -, la "gouvernance" est surtout faite de mécanismes liés au marché en s'appuyant sur des partenariats importants entre secteurs public et privé (les fameux "PPP").

On considère qu'un système de décision publique peut s'appeler “gouvernance” lorsqu'il comprend les éléments suivants :

- la participation des intéressés - les fameux "stakeholders" des Anglo-Saxons - à l'élaboration et à l'application ultérieure des décisions concrètes ; on pense aujourd'hui que le système de représentation par les élus n'est efficace pour promouvoir la démocratie que s'il est complété par des dispositifs d'implication des intéressés et du public dans les décisions qu'ils prennent, et ceci est particulièrement le cas dans des thématiques comme la santé et l'environnement (dont la forêt) ;

- l'articulation entre décisions prises aux niveaux international, national, et local (régional, départemental) ; en effet, le débat sur l'évolution des centres de décision publique tend à donner certes plus de poids au niveau international, mais également au niveau local, comme une sorte de contre-pouvoir, mais aussi parce que la participation précédemment citée suppose un rapprochement géographique net entre autorité publique et intéressés (d'où l'importance du local) ;

- le caractère intersectoriel de l'approche de tout problème ; si jusqu'ici en effet, on traitait de la forêt comme d'un problème séparé des autres, la discussion sur le développement durable a amené à le lier intimement aux questions centrales de l'environnement, de l'agriculture et du développement rural, ainsi qu'avec celle de l'aménagement du territoire ; aujourd'hui, la façon efficace de traiter la forêt est d'en faire un élément important de ces problématiques jusqu'ici considérées comme périphériques ;

- l'importance de l'expertise sociale et scientifique, et non plus seulement technique, comme guide des décisions ; aujourd'hui, les experts participant au débat confrontent leurs différentes approches et solutions d'une façon transparente, sans qu'existe une seule solution technique présentée comme inéluctable ;

- le fait qu'aucune décision n'est a priori irrévocable, car soumise aux appréciations des acteurs dans un contexte économique et social de changement permanent, amène enfin à instituer des systèmes de suivi et d'évaluation, alors que le secteur forestier était bien connu pour sa faiblesse dans ce domaine. 


\section{OÙ EN EST-ON EN FRANCE ?}

La France, ni plus ni moins que les autres pays qui tous sont concernés au même titre, a déjà traduit dans les faits certaines évolutions dessinées au plan international.

Le rapport Bianco d'abord, puis la promotion de PEFC et le vote de la loi d'orientation sur la forêt de 2001 ont incontestablement marqué des étapes importantes dans l'évolution des modes de gestion et de gouvernance.

Les chartes forestières de territoire, mises en place depuis les années 2000, constituent une innovation institutionnelle fondamentale ; elles substituent en effet à une politique du guichet (où les stakeholders se voient attribuer a priori par la réglementation leurs droits - notamment au financement - et devoirs), une logique du projet, où des actions conjointes à plusieurs acteurs locaux sont supposées donner un contenu participatif et construit au développement local (cf. l'article de Patrick Moquay).

Basées sur l'idée que le développement forestier ne se décrète pas d'en haut mais se construit sur le terrain, dans le dialogue et la participation de toutes les parties intéressées, les chartes forestières de territoire constituent un cadre simple et souple pour l'élaboration de projets partagés axés sur la forêt et le bois. À ce jour, 76 chartes forestières de territoire couvrant une surface totale de 5700000 ha et une surface forestière de 2500000 ha sont en activité ou en cours d'élaboration. L'expérience a permis d'identifier différentes clés de succès faute desquelles la charte reste peu productive : un périmètre cohérent, une ou des problématiques bien identifiées, la mobilisation de tous les acteurs, une structure porteuse garantissant une animation pérenne, une méthode rigoureuse de travail collectif, des moyens financiers et surtout un portage politique.

Les communes forestières ont sans doute eu un rôle moteur dans ce mouvement; d'abord elles ont souhaité mieux affirmer leur rôle de propriétaire forestier et donc de décideur des grands choix engageant le devenir de la forêt communale. Mais surtout, conjuguant leur responsabilité forestière et leur compétence de développement local, elles ont compris que l'élaboration des projets forestiers devait partir du niveau local : la création des chartes forestières de territoire par la loi forestière de 2001 a constitué une réponse adéquate dans laquelle les élus des communes forestières, forts de leur statut d'élu du suffrage universel, se sont naturellement investis.

Des progrès sont par ailleurs en cours de réalisation en matière de formulation de plans d'aménagement intégrés, notamment en zone de montagne, même si en France on reste encore loin des changements apportés dans les approches de l'aménagement dans d'autres pays, où il est vrai que l'aspect normatif est moins net et la prise en compte du contexte environnemental plus déterminante que chez nous (cf. l'article de Rodolphe Schlaepfer).

À l'échelle plus large du massif de montagne, le Comité interministériel pour l'aménagement du territoire (CIAT) "rural" de septembre 2003 a lancé un travail expérimental d'élaboration de deux schémas stratégiques forestiers de massif dans les Alpes et le Massif central : le résultat a été suffisamment probant pour que la loi d'orientation agricole du 5 janvier 2006 élargisse la mesure à l'ensemble des massifs montagneux et un troisième schéma est entrepris dans les Pyrénées.

C'est peut-être en matière de participation que l'évolution, fortement dépendante des facteurs culturels et historiques, est la plus lente (cf. l'article d'Irina Kouplevatskaya). Mais on peut noter tout de même que les nouveaux outils (dont les chartes forestières de territoire) poussent à des solutions de plus en plus concertées, et qu'une demande émanant des acteurs de terrain se fait jour pour disposer d'un appui en la matière. 
Même si des progrès ont été réalisés dans le dialogue avec les organisations de défense de l'environnement, leurs représentants se plaignent que leurs positions ne soient pas prises en compte dans les décisions finales (cf. l'article d'Emmanuelle Neyroumande). Toutefois, là encore, les choses semblent évoluer également ; il n'est que de constater que le récent Grenelle de l'environnement a donné lieu à des discussions qui, plus que ce n'était le cas dans les années précédentes, peuvent conduire à une véritable négociation de solutions communes. Exemple particulièrement significatif : une plateforme commune a été établie entre forestiers privés et publics et France Nature Environnement : sous le titre " produire plus de bois tout en préservant mieux la biodiversité - une démarche territoriale concertée dans le respect de la gestion multifonctionnelle ", ce texte affirme clairement que production et protection peuvent aller de pair et retient différentes pistes pour assurer une étroite cohérence entre les politiques de mobilisation raisonnée du bois et de préservation de la biodiversité. Ce qui paraissait inconciliable il y a encore peu de temps est aujourd'hui affirmé tranquillement! Restera à faire vivre cet accord, ce ne sera sûrement pas toujours facile, mais le dialogue est installé qui sera mené à la fois au niveau national et au niveau local en privilégiant l'échelle du territoire de projet.

Quoi qu'il en soit, la question de la gestion durable des forêts, désormais bien acceptée et digérée en France, reste structurante des évolutions en cours (cf. l'article de Christian Barthod).

\section{L'EXEMPLE DU PARTENARIAT COMMUNES FORESTIÈRES - ONF}

L'échelon local de prise de décision publique, en symétrique du niveau international, s'est trouvé lui aussi largement promu par les évolutions récentes des modes de gouvernance, qui s'inscrivent dans un contexte général de décentralisation des choix publics (cf. l'article de Michel Badré).

L'évolution des relations entre les communes forestières et l'Office national des Forêts fournit un exemple frappant de ces changements.

La loi d'orientation sur la forêt de juillet 2001 a banni, on s'en souvient, le mot "soumis " du vocabulaire forestier : une forêt communale, dorénavant, "relève du régime forestier ", elle n'est plus soumise au régime forestier ; et certes les maires ne sont plus soumis - s'ils l'ont jamais été - à l'ONF! Dans la logique de la décentralisation, les communes forestières ont voulu pleinement assurer leur rôle de propriétaire forestier, déterminer les grands objectifs de la forêt et fixer les grands choix de gestion à travers l'aménagement forestier et le programme annuel de coupes et de travaux. En somme, au maire les grandes décisions, à l'ONF la fonction de conseil et de mise en œuvre du régime forestier.

La charte de la forêt communale signée le 16 octobre 2003 entre l'ONF et la Fédération nationale des Communes forestières (FNCOFOR) traduit cette évolution : " Il revient au conseil municipal et au maire de gérer et d'administrer le patrimoine forestier communal dans toutes ses dimensions. Cette gestion et cette administration se réalisent dans le cadre du régime forestier dont l'Office national des Forêts est chargé de la mise en œuvre ». Et cette charte précise clairement les rôles et responsabilités respectifs et complémentaires des collectivités et de l'ONF.

Un tel texte est évidemment un document vivant. Il a déjà été complété une première fois deux ans plus tard, en septembre 2005 , pour créer une instance partenariale de pilotage de la politique de commercialisation des bois communaux et de développement de la mise en place des contrats d'approvisionnement. Dans la période de bouleversement du marché du bois et de chute des recettes de vente de bois communaux qui a suivi les tempêtes de décembre 1999, il est apparu en effet que les élus devaient intervenir en direct dans le registre de la commercia- 
lisation des bois, décider avec l'ONF et en appuyer les actions : le comité des ventes des bois communaux se réunit chaque mois dans ce but à Paris à la satisfaction partagée de l'Office et des communes.

Sur le même modèle et face à une autre question-clé pour les élus, celle des travaux forestiers, un comité national des travaux a été créé en septembre 2006 : sa mission est de rechercher les moyens de maintenir le niveau des travaux en forêt communale à la hauteur des enjeux de la gestion durable des forêts au meilleur prix et en répondant au mieux aux besoins des communes forestières.

Ce partenariat " vivant, adulte, confiant et attentif " entre les communes forestières et l'Office national des Forêts s'est concrétisé, acte particulièrement symbolique et fort, par l'apposition de la signature du président de la Fédération nationale des Communes forestières au bas du contrat État-ONF 2007/2011.

Écho direct aux thèmes du changement et de la gouvernance dont il a été longuement question à Tignécourt, les politiques territoriales de la forêt entrent maintenant dans le champ des discussions partenariales entre Office et communes forestières pour définir le rôle et la place de chacun : pour parler simplement, on pourrait résumer ainsi la position des communes forestières : aux élus le portage, à l'ONF le soutien.

Le rôle et les responsabilités des élus locaux ne cessent donc de s'affirmer au carrefour des représentations des intérêts directs et du public en général, c'est-à-dire des citoyens.

\section{LES QUESTIONS EN DÉBAT}

L'évolution mentionnée n'est certainement pas arrivée à son terme, et dans la façon dont les équilibres s'établissent, des questions restent entières.

Quel type de participation promouvoir en France, et à quel niveau ? Jusqu'à présent, la culture nationale et l'histoire des institutions forestières avaient promu une consultation sélective d'acteurs directement engagés dans la production de bois. Mais la territorialisation des actions forestières amène autour de la table de nouveaux acteurs (élus locaux, représentants d'associations) qui jouent un rôle majeur dans la définition d'actions multifonctionnelles. Est-ce le temps, au moins au niveau local, de construire de véritables processus de concertation entre acteurs ?

Il est encore un peu tôt pour prédire ce que sera le mode de fonctionnement des chartes forestières de territoire dans les prochaines années. Mais si la formule s'avérait efficace et soutenue par l'Union européenne, à quels mécanismes économiques devra-t-on réfléchir pour porter un développement local équilibré ?

La place de plus en plus importante occupée par les questions environnementales et énergétiques ne porte-t-elle pas à reconsidérer plus globalement la façon avec laquelle on doit concevoir la satisfaction des utilités apportées par la forêt ? Le modèle, classique en France, de l'effet de sillage de la production de bois, c'est-à-dire l'idée selon laquelle la production de matière ligneuse seule permet, par la valeur qui lui est donnée sur le marché, de financer les autres fonctions, est-il encore tenable, et si non par quoi le remplacer dans un contexte social où protection, récréation et services environnementaux tiennent une place de plus en plus grande et où l'opinion du public se forme et s'exprime à la fois à travers les média ?

Et puisqu'on parle de financement, quels mécanismes économiques, probablement liés au marché, doit-on inventer pour que les acteurs forestiers, qui contribuent au maintien de fortes externalités 
positives dont la société et l'économie bénéficient, puissent être au moins partiellement rémunérés pour cette fonction collective?

Dans le passé, le Fonds forestier national doté d'un budget annuel moyen de l'ordre de 100 millions d'euros a permis de mener une action volontariste de grande qualité en faveur du développement forestier. La forêt est en mesure aujourd'hui, le "Grenelle" le reconnaît, d'apporter une contribution déterminante aux grands enjeux de notre civilisation, la préservation de la biodiversité et la lutte contre les changements climatiques pour n'en citer que deux ; quels instruments financiers, de hauteur comparable à ceux du FFN, seront mis en place pour permettre le saut quantitatif et qualitatif qu'impose cette ambition ?

Une question centrale est bien en effet de savoir quelles seront les incidences concrètes locales en matière de choix publics du débat international sur le changement climatique, qui s'est désormais posé comme cadre aux discussions sur la gestion durable des forêts, et qui peut modifier largement les modalités de la gestion forestière et ainsi redistribuer les cartes entre acteurs du développement forestier (cf. l'article de Bernard Roman-Amat) ?

Et que dire des nouvelles orientations que cette évolution globale du contexte dans lequel s'inscrivent les actions forestières impose en matière d'enseignement et de recherche, sinon qu'il y a tout à parier que les forestiers de demain devront exercer différemment leurs fonctions, et donc disposer d'un nouveau bagage de connaissances auquel il faut songer dès maintenant (cf. article de Patrice Harou et Christophe Voreux).

Toutes ces questions donnent une idée du vaste débat qui va se poursuivre au cours des prochaines années, dans un contexte fait d'accélération des changements (cf. l'article conclusif de Gérard Buttoud).

Cette livraison de la Revue forestière française n'a pas la prétention de trancher ce débat, mais plus simplement, en abordant certaines de ces interrogations, d'aider les acteurs forestiers à mieux comprendre les évolutions en cours, et de leur permettre ainsi de mieux situer leur rôle et leur action.

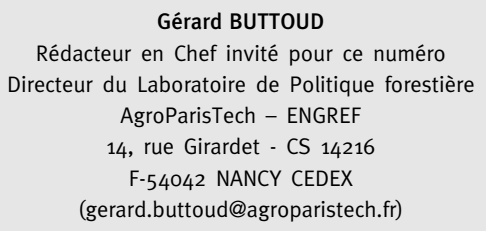

\section{Jean-Claude MONIN \\ Président délégué de \\ la FÉDÉRATION NATIONALE}

DES COMMUNES FORESTIÈRES (FNCOFOR)

${ }_{1}$ er Vice-Président de l'ONF

13, rue Général Bertrand

F-75007 PARIS 